\title{
Oligodendrocyte Specification in Zebrafish Requires Notch- Regulated Cyclin-Dependent Kinase Inhibitor Function
}

\author{
Hae-Chul Park, Janene Boyce, Jimann Shin, and Bruce Appel \\ Department of Biological Sciences, Vanderbilt University, Nashville, Tennessee 37235-1634
}

Cyclin-dependent kinase inhibitors (Cdkis) influence both cell-cycle progression and differentiation of neural cells. However, the precise roles of Cdkis in coordinating formation of neurons and glia and the mechanisms that regulate expression of genes that encode Cdkis in the vertebrate CNS remain unknown. Here, we report that, in zebrafish, expression of the Cdki gene cyclin-dependent kinase inhibitor 1c ( $c d k n 1 c)$, a $p 57$ homolog, is negatively regulated by Delta-Notch signaling and that Cdkn1c function is required for neural plate cells to stop dividing and differentiate as neurons on schedule, even in the absence of Notch signaling activity. Furthermore, Cdknlc function is required for specification of oligodendrocytes from ventral spinal cord precursors. We propose that levels of $c d k n 1 c$ expression are an important factor in regulating neural development: high levels of Cdknlc promote cell-cycle exit and neuronal development, whereas, during late embryogenesis, neural cells that have low but functional levels of Cdkn1c, regulated by Notch activity, are specified for oligodendrocyte fate.

Key words: oligodendrocyte; cell cycle; cell fate; neural precursor; neurogenesis; spinal cord

\section{Introduction}

During vertebrate neural development, proliferative precursor cells produce different types of postmitotic neurons at different times, revealing a correlation between the time at which a precursor stops dividing and neuronal identity. A particularly good illustration of this was provided by experiments in which cerebral cortex precursors were transplanted from young donors to older hosts (McConnell and Kaznowski, 1991). Remarkably, precursors that remained proliferative at the time of transplantation adopted fates appropriate for the host, whereas those that stopped dividing before transplantation retained donor identity. Thus, specification of multipotent cortical precursors for particular neuronal identities apparently occurred at about the time of cell-cycle exit. Cell-cycle control, therefore, might be an essential feature of mechanisms that specify neuronal fate.

Timing also seems to be important for glial development because, in general, glia are produced after neurons. However, for oligodendrocytes, the myelinating cell type of the CNS, the relationship between the proliferative status of a neural precursor and specification for oligodendrocyte fate is not clear. In particular, cells fated to give rise to oligodendrocytes do not stop divid-

Received March 13, 2005; revised June 10, 2005; accepted June 14, 2005.

This work was supported by the National Multiple Sclerosis Society and National Institutes of Health (NIH) Grant NS46668. We thank Lee Anne Givan and Tarek Fakouri for initiating these experiments and Dr. Bruce Carter for critical comments on this manuscript. The GAL4/UAS transgenic lines were developed in the laboratory of J. CamposOrtega. The anti-BrdU antibody, developed by S. J. Kaufman, was obtained from the Developmental Studies Hybridoma Bank, developed under the auspices of the National Institute of Child Health and Human Development and maintained by The University of lowa, Department of Biological Sciences (lowa City, IA). Confocal microscopy was performed using equipment made available by the Vanderbilt University Medical Center Cell Imaging Core Resource, supported by NIH Grants 1S10RR15682, CA68485, and DK20593.

Correspondence should be addressed to Dr. Bruce Appel, Department of Biological Sciences, VU Station B, Box 35-1634, Vanderbilt University, Nashville, TN 37235-1634. E-mail: b.appel@vanderbilt.edu.

DOI:10.1523/JNEUROSCI.0981-05.2005

Copyright $\odot 2005$ Society for Neuroscience $\quad$ 0270-6474/05/256836-09\$15.00/0 ing but exist as proliferative oligodendrocyte progenitor cells (OPCs), which have characteristic behaviors, morphologies, and gene expression profiles (Baumann and Pham-Dinh, 2001; Miller, 2002). Eventually, many OPCs stop dividing and differentiate as myelinating oligodendrocytes, whereas others apparently persist into adulthood (Levison et al., 1999; Horner et al., 2000). Here, cell-cycle control influences the balance of immature OPCs and myelinating oligodendrocytes.

Molecules that likely play widespread roles in the timing of neuronal and glial development include cyclin-dependent kinase inhibitors (Cdkis). Cdkis negatively regulate complexes composed of cyclins and cyclin-dependent kinases (Cdks), which promote progression through the cell cycle (Cunningham and Roussel, 2001). For instance, cyclin E assembles with cdk2 to drive the $G_{1}$ to $S$ phase transition. Cdki proteins of the Cip/Kip class, which include p $21^{\mathrm{Cip} 1}, \mathrm{p} 27^{\mathrm{Kip} 1}$, and p $57^{\mathrm{Kip} 2}$, bind to cyclin E-cdk2 in a 1:1 stoichiometry and prevent entry into $S$ phase. Although various experimental strategies provided evidence that Cdki functions promote formation of postmitotic neurons and regulate proliferation and differentiation of OPCs (CasacciaBonnefil et al., 1997, 1999; Durand et al., 1998; Zezula et al., 2001; Carruthers et al., 2003; Vernon et al., 2003), the mechanisms that control Cdki expression in the developing CNS and whether Cdki function is necessary to specify neural precursors for oligodendrocyte fate have not been fully explored.

This report describes a series of loss- and gain-of-function experiments designed to investigate regulation of $c d k i$ gene expression and how $c d k i$ function influences the timely formation of neurons and oligodendrocytes. Our results show that, in zebrafish, Notch signaling limits the number of neural cells that express cyclin-dependent kinase inhibitor $1 c(c d k n 1 c)$, and $c d k n 1 c$ function is necessary for production of early born neurons at the appropriate time. Surprisingly, we also learned that $c d k n 1 c$ func- 
tion is necessary for OPC specification from neural precursors, thereby expanding the previously described roles of Cdkis in oligodendrocyte development.

\section{Materials and Methods}

Fish breeding and maintenance. Embryos were produced by pairwise matings, raised at $28.5^{\circ} \mathrm{C}$ in egg water or embryo medium (EM) (Westerfield, 2000), and staged according to hours postfertilization (hpf), days postfertilization (dpf), and morphological criteria (Kimmel et al., 1995). mib ${ }^{\text {ta52b }}$ mutant fish (Jiang et al., 1996; Itoh et al., 2003), $\mathrm{Tg}$ (hsp70:GAL4) and $\operatorname{Tg}\left(\right.$ UAS:Notch $\left.1 a^{a c}-m y c\right)$ fish (Scheer and Campos-Ortega, 1999), $\operatorname{Tg}$ (olig2:egfp) fish (Shin et al., 2003; Park et al., 2004), and $\operatorname{Tg}(h s p 70$ : $X d n S u(H) m y c$ ) fish (Latimer et al., 2005) were used for this study.

Generation of $\operatorname{Tg}(\mathrm{hsp} 70: \mathrm{cdkn} 1 \mathrm{c}-\mathrm{myc})$ transgenic fish. To produce $\mathrm{Tg}$ (hsp70:cdknlc-myc) fish, we first exchanged the cytomegalovirus promoter of pCS2 2 MT (Turner and Weintraub, 1994) for the zebrafish heat shock 70 (hsp70) promoter (Shoji et al., 1998) to produce pCS2hsp70+MT. Next, we inserted $c d k n 1 c$ cDNA sequence so that it created a reading frame fusion to sequence encoding the Myc epitope tag at the $3^{\prime}$ end of $c d k n 1 c$, creating pCS2hsp70:cdkn1c-myc. We then transferred a fragment containing hsp70:cdkn1c-myc and simian virus 40 poly(A) between the two I-SceI recognition sequences of pBSI2, creating pBShsp70:cdkn1c-mycI2. Transgenic lines were obtained essentially as described previously (Thermes et al., 2002). The final plasmid was injected into one-cell stage embryos at a concentration of $30 \mathrm{ng} / \mu \mathrm{l}$ with 1 $\mathrm{U} / \mu \mathrm{l} I$-SceI (New England Biolabs, Beverly, MA), $0.5 \times I$-SceI buffer, and $0.05 \%$ phenol red. Injected fish were raised to adulthood and crossed to wild type. To identify transgenic embryos and, consequently, germ-linetransformed founders, we heat shocked embryos by incubating them at $40^{\circ} \mathrm{C}$ for $30 \mathrm{~min}$ and, after a recovery period, fixed them and performed anti-Myc immunocytochemistry. To establish stable lines, we again mated germ-line-transformed founders to wild-type fish, raised the embryos to adulthood, and repeated the Myc immunocytochemistry screen.

Bromodeoxyuridine labeling, in situ RNA hybridization, and immunocytochemistry. Manually dechorionated embryos were labeled with bromodeoxyuridine (BrdU) by incubating them for $20 \mathrm{~min}$ on ice in a solution of $10 \mathrm{~mm}$ BrdU and 15\% DMSO in EM. The embryos were then placed in $\mathrm{EM}$ and incubated $20 \mathrm{~min}$ at $28.5^{\circ} \mathrm{C}$ and fixed using $4 \%$ paraformaldehyde in PBS. Embryos were processed for in situ RNA hybridization to detect $c d k n 1 c$ message, treated $1 \mathrm{~h}$ with $2 \mathrm{M} \mathrm{HCl}$, and then processed for anti-BrdU immunocytochemistry.

In situ RNA hybridization data for $c d k n 1 b$ were retrieved from the Zebrafish Information Network (ZFIN), the Zebrafish International Resource Center, University of Oregon [Eugene, OR; http://zfin.org/ (December 19, 2004)]. In situ RNA hybridization to detect $c d k n 1 c$, isl1, isl2, and $p l p / d m 20$ expression was performed as described previously (Hauptmann and Gerster, 2000). For detection of $c d k n 1 c$ expression in $\mathrm{Tg}$ (olig2: egfp) embryos, in situ hybridization was performed on cryosections with no proteinase $\mathrm{K}$ treatment to preserve enhanced green fluorescent protein (EGFP) fluorescence.

For immunocytochemistry, we used the following primary antibodies: mouse anti-BrdU (G3G4; 1:1000; Developmental Studies Hybridoma Bank, Iowa City, IA), anti-c-Myc (Ab-1; 1:100; Oncogene Research Products, Cambridge, MA), mouse anti-HuC/D (1:20; Molecular Probes, Eugene, OR), and rabbit anti-Sox10 (1:1000), which we raised against the peptide sequence DGGKTQIKSETHFPGD using a commercial service (Open Biosystems, Huntsville, AL). For fluorescent detection of antibody labeling, we use Alexa Fluor 568 goat anti-mouse or goat anti-rabbit conjugates (1:500; Molecular Probes). Embryos were sectioned as described previously (Park and Appel, 2003). In situ hybridization images were collected using a QImaging (Burnaby, British Columbia, Canada) Retiga Exi color CCD camera mounted on a compound microscope and imported into Adobe Photoshop (Adobe Systems, San Jose, CA). Joint in situ hybridization and fluorescence images were collected separately and combined using Photoshop. Image manipulations were limited to levels, curve, hue, and saturation adjustments. Fluorescence images were collected using a Zeiss (Oberkochen, Germany) LSM510 laser scanning confocal microscope.
Heat-induced gene expression. To induce expression of constitutively active Notchla, embryos were collected from matings of $\mathrm{Tg}(\mathrm{hsp} 70$ : GAL4) and $\operatorname{Tg}$ (UAS:Notch $\left.1 a^{a c}-m y c\right)$ adults and raised at $28.5^{\circ} \mathrm{C}$. At 7.5 hpf, embryos were transferred to $\mathrm{EM}$ at $39.0^{\circ} \mathrm{C}$ for $30 \mathrm{~min}$ and then returned to $28.5^{\circ} \mathrm{C}$ until the three-somite stage ( $11 \mathrm{hpf}$ ). Approximately one-fourth of the embryos should inherit both transgenes, which we confirmed by anti-Myc immunocytochemistry (data not shown). To induce expression of Cdkn1cMyc or a Myc-tagged dominantnegative DNA-binding mutant form of frog Suppressor of Hairless $[\mathrm{dnSu}(\mathrm{H}) \mathrm{Myc}]$, we intercrossed $\operatorname{Tg}($ hsp 70:cdkn1c-myc) or $\operatorname{Tg}(h s p 70$ : $X d n S u(H) m y c)$ adults, respectively, raised embryos at $28.5^{\circ} \mathrm{C}$, transferred them to $\mathrm{EM}$ at $40^{\circ} \mathrm{C}$ for $30 \mathrm{~min}$, and continued incubation at $28.5^{\circ} \mathrm{C}$ until appropriate stages for fixing.

Morpholino injections. An antisense morpholino oligonucleotide (MO) having the sequence 5'-TGCCATGATGTCTAAAAGTCAATGC-3' (Gene Tools, LLC, Philomath, OR) was designed to target the $c d k n 1 c 5^{\prime}$-untranslated region sequence and translation start site. The morpholino oligonucleotide was dissolved in $1 \times$ Danieau solution at a concentration of $20 \mu \mathrm{g} / \mathrm{ul}$ and further diluted with distilled water. Two nanograms of morpholino oligonucleotide were injected into one- to two-cell-stage embryos. For control experiments, a similar amount of a standard control MO having the sequence 5 '-CCTCTTACCTCAGTTACAATTTATA- $3^{\prime}$ was injected. To test the ability of $c d k n 1 c$ MO to block Cdkn1c expression, we injected MO into embryos produced by $\mathrm{Tg}$ (hsp70:cdkn1c-myc) adults and heat shocked embryos at $7.5 \mathrm{hpf}$ by incubating them at $38^{\circ} \mathrm{C}$ for $30 \mathrm{~min}$, fixed them at $11 \mathrm{hpf}$, and processed them for anti-Myc immunocytochemistry.

\section{Results}

\section{Identification of zebrafish $c d k n 1 c$}

We identified zebrafish expressed sequence tags (ESTs) with sequence similarity to $p 27$ and $p 57$ genes of other vertebrate species using basic local alignment search tool (Altschul et al., 1990). These ESTs were derived from genes now known as $c d k n 1 b$ and $c d k n 1 c$, respectively (Sprague et al., 2001). We did not find ESTs with homology to $p 21$. Whole-mount in situ RNA hybridization detected no $c d k n 1 b$ expression during early stages of neural development (data not shown, but see ZFIN, http://zfin.org/cgibin/webdriver?MIval = aa-xpatview.apg\&OID = ZDB-XPAT030521-5). In contrast, neural plate and spinal cord cells expressed $c d k n 1 c$ (Figs. 1, 2,4). Notably, the neural plate expression of $c d k n 1 c$ is like that of $p 27^{X i c 1}$ of Xenopus laevis and Xenopus tropicalis (Hardcastle and Papalopulu, 2000; Carruthers et al., 2003; Vernon et al., 2003). Thus, Cdkn1c may regulate early neural development in zebrafish similarly to $\mathrm{p} 27^{\mathrm{Xic1}}$ in frogs.

\section{Notch signaling regulates $c d k n 1 c$ expression}

We showed previously that Notch signaling is required to limit the formation of early born primary neurons and maintain proliferative neural precursors (Appel et al., 2001; Park and Appel, 2003). Because $p 27^{X i c 1}$ promotes formation of neurons within the neural plate of frogs (Carruthers et al., 2003; Vernon et al., 2003), we speculated that maintenance of neural precursors by Notch is mediated by transcriptional repression of $c d k n$ genes. Therefore, we compared the distribution of $c d k n 1 c$ transcripts between embryos that had different levels of Notch activity. In wild-type embryos, neural plate cells expressed $c d k n 1 c$ RNA in a mosaic pattern, consistent with the pattern of the earliest born primary neurons, and adaxial mesoderm cells expressed $c d k n 1 c$ uniformly at high level (Fig. 1A,D). Cells that expressed $c d k n 1 c$ did not incorporate the thymidine analog BrdU, a marker of S-phase cells, which supports the idea that nonproliferative cells express cdkn1c (Fig. 3G). In both $d l a^{-/-}$; $d l d^{-/-}$embryos, which lack functions of the DeltaA and DeltaD ligands (Appel et al., 1999; Holley et al., 2000), and mib ${ }^{-/-}$embryos, which are deficient for 
an E3 ubiquitin ligase necessary for efficient Notch signaling activity (Itoh et al., 2003), excess neural plate cells expressed $c d k n 1 c$ RNA, but adaxial expression appeared normal (Fig. $1 B, C, E$ ). This is consistent with formation of excess primary neurons in these mutant embryos (Jiang et al., 1996; Schier et al., 1996; Appel et al., 2001; Itoh et al., 2003). In contrast, forced expression of a constitutively active form of Notch1a (Scheer and Campos-Ortega, 1999; Scheer et al., 2001, 2002) just before the beginning of neurogenesis concomitantly inhibited neural plate $c d k n 1 c$ expression (Fig. $1 F$ ) and formation of primary neurons (Fig. 1G,H) but did not affect adaxial cell $c d k n 1 c$ expression (Fig. $1 F)$. Thus, Notch signaling regulates $c d k n 1 c$ expression in neural plate but not adaxial mesoderm cells.

We next asked whether Notch activity continuously limits $c d k n 1 c$ expression and neurogenesis by using the transgenic line $\mathrm{Tg}(h s p 70: \mathrm{XdnSu}(H) m y c)$ (Latimer et al., 2005) to express $\mathrm{dnSu}(\mathrm{H}) \mathrm{Myc}$ (Wettstein et al., 1997) under transcriptional control of the zebrafish $h s p 70$ promoter (Shoji et al., 1998). At 36 hpf, control embryos expressed $c d k n 1 c$ RNA in columns of spinal cord cells that were just outside the proliferative cells that occupy the medial spinal cord (Fig. 2A). Similarly staged embryos heat shocked at $24 \mathrm{hpf}$ to induce $\operatorname{dnSu}(\mathrm{H}) \mathrm{Myc}$ expressed $c d k n 1 c$ at high level throughout the spinal cord (Fig. 2B), consistent with our observations that Notch signaling inhibits $c d k n 1 c$ expression in neural plate cells. To examine the effect of this treatment on formation of neurons and oligodendrocytes, we repeated the experiment using $\operatorname{Tg}($ olig2:egfp); $\operatorname{Tg}(h s p 70: X d n S u(H)$ $m y c$ ) embryos, in which olig2:EGFP expression marks ventral spinal cord precursors that give rise to motor neurons, interneurons, and oligodendrocytes (Shin et al., 2003; Park et al., 2004). All olig2: $\mathrm{EGFP}^{+}$cells developed as neurons, with a concomitant loss of proliferative precursors (Fig. 2D) and no OPCs formed (Fig. 2F). Thus, Notch signaling is required throughout the period of motor neuron production to inhibit $c d k n 1 c$ expression and maintain a pool of precursors that can give rise to OPCs. These data establish that, in zebrafish, Notch activity negatively regulates $c d k n 1 c$ expression and support the possibility that Notch inhibition of cell-cycle exit and primary neurogenesis and maintenance of spinal cord precursors occurs, at least in part, via inhibition of $c d k n 1 c$.

Loss of $c d k n 1 c$ function increases neural cell proliferation and delays primary neuron production

To investigate $c d k n 1 c$ function, we designed an antisense MO to block translation of $c d k n 1 c$ mRNA. We tested the effectiveness of our $c d k n 1 c$ MO by injecting it into $\mathrm{Tg}(h s p 70: c d k n 1 c-m y c)$ embryos, which express Myc epitope-tagged Cdkn1c under control of the heat shock 70 promoter (Shoji et al., 1998). The $c d k n 1 c \mathrm{MO}$ has sequence complementary to the transcript expressed from the transgene and should inhibit its translation. Indeed, $c d k n 1 c$ MO-injected embryos expressed Cdkn1cMyc at much lower levels than noninjected embryos (Fig. $3 A, B$ ).
If Cdkn1c promotes cell-cycle exit and primary neurogenesis, embryos that lack Cdkn1c function should have a deficiency of primary neurons. At neural plate stage, $80.2 \%$ (325 of 405) of $c d k n 1 c$ MO-injected embryos had few primary neurons (compare Figs. $3 C, 1 G$ ). This is consistent with the possibility that Notch inhibition of $c d k n 1 c$ expression prevents formation of neurons in the zebrafish neural plate. To further establish the relationship between Notch signaling and Cdkn1c function, we injected embryos produced by intercrosses of $\mathrm{mib}^{+/-}$adults with control or $c d k n 1 c$ MOs. Twenty-six percent (43 of 165) of embryos injected with control MO produced excess primary neurons, close to the expected frequency of $25 \%$ homozygous mutant embryos (data not shown). In contrast, $12.6 \%$ (20 of 159) of embryos injected with $c d k n 1 c$ MO formed groups of densely spaced neurons evident of a mib mutant phenotype, although, overall, these embryos had deficits of primary neurons (Fig. 3D). Most remaining embryos had few neurons, similar to wild-type embryos injected with $c d k n 1 c \mathrm{MO}$ (data not shown). Thus, loss of $c d k n 1 c$ function suppressed the excess primary neuron phenotype of $\mathrm{mib}^{-/-}$embryos, suggesting that Notch-signalingdeficient embryos produce excess primary neurons because they fail to limit $c d k n 1 c$ expression to subsets of neural plate cells.

In mice, flies, and nematodes, cells that were deficient for Cdki activity underwent additional divisions but ultimately exited the cell cycle and differentiated (de Nooij et al., 1996; Lane et al., 1996; Hong et al., 1998; Lowenheim et al., 1999; Levine et al., 2000; Miyazawa et al., 2000; Fukuyama et al., 2003). Frog em- 


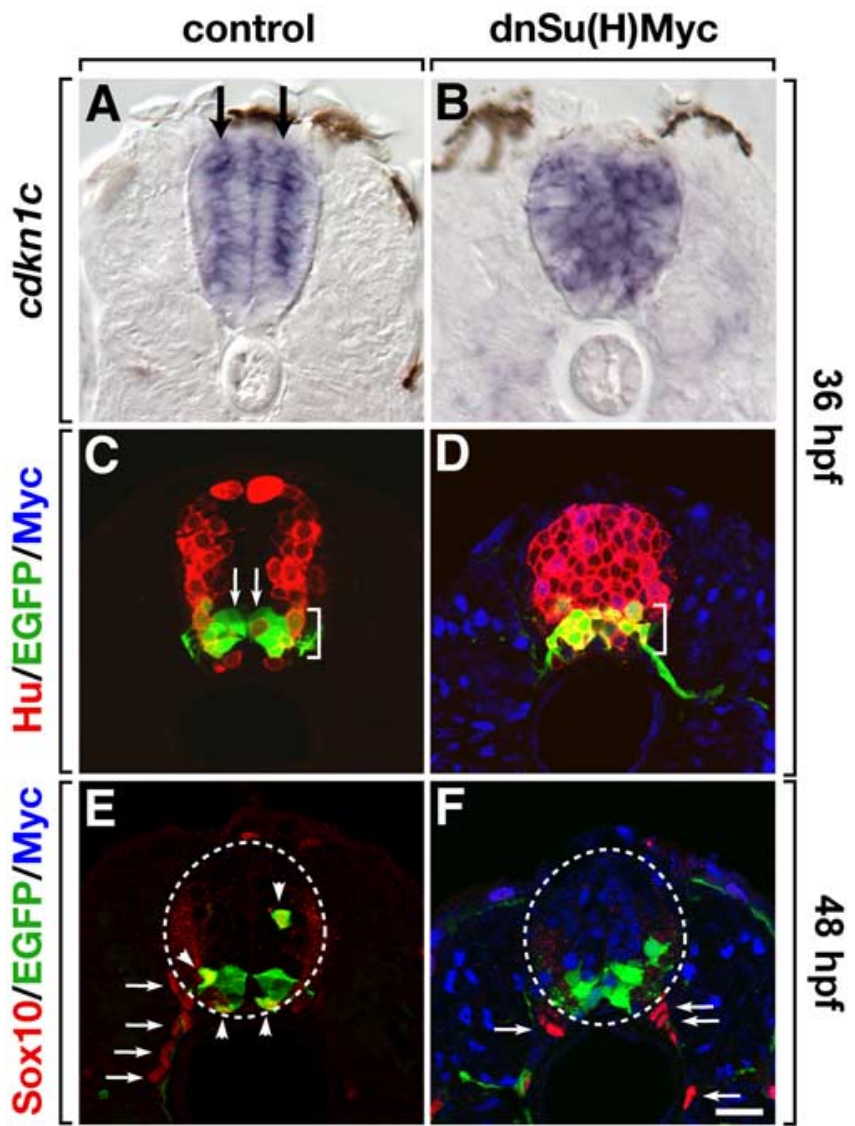

Figure 2. Notch signaling is required continuously during early neural development to inhibit $c d k n 1 c$ expression and neurogenesis. All panels are transverse sections with dorsal up. $A$, Control embryo (36 hpf) expressed cdkn1c at a high level in two columns (arrows) of cells located between the ventricle and pial surface of the spinal cord. B, Spinal cord cells of $\operatorname{Tg}$ (hsp 70: $\mathrm{XdnSu}(\mathrm{H}) \mathrm{my}$ c) embryo heat shocked at $24 \mathrm{hpf}$ uniformly expressed cdkn $1 \mathrm{c}$ at a high level at 36 hpf. C, D, Embryos (36 hpf) labeled with Hu antibody to reveal neurons (red fluorescence). C, Control Tg(olig2:egfp) embryo. EGFP ${ }^{+}, \mathrm{Hu}^{+}$neurons (green cells outlined by yellow within ventral spinal cord indicated by bracket) and EGFP ${ }^{+}, \mathrm{Hu}^{-}$precursor cells in the ventral, medial spinal cord (green cells indicated by arrows) are evident. D, Tg(olig2:egfp); $T g(h s p 70: X d n S u$ (H)myc) embryo heat shocked at 24 hpf. All EGFP ${ }^{+}$cells were $\mathrm{Hu}^{+}$neurons (green cells outlined by yellow). Blue labeling reveals antibody labeling of Myc epitope-tagged transgene. $\boldsymbol{E}, \boldsymbol{F}$, Embryos ( $48 \mathrm{hpf}$ ) labeled with Sox 10 antibody to label OPCS. Spinal cords are outlined by dashed circles. The arrows indicate Sox $10^{+}$Schwann cells outside the spinal cord. $\boldsymbol{E}$, Control Tg(olig2: egfp) embryo showing several EGFP ${ }^{+}$, Sox $10^{+}$OPCs (yellow cells marked by arrowheads). $\boldsymbol{F}$, Tg(olig2:egfp); $\operatorname{Tg}(h s p 70: X d n S u(H) m y c)$ embryo heat shocked at $24 \mathrm{hpf}$ had no Sox $10^{+}$OPCs. Scale bar: (in $\boldsymbol{F}) 20 \mu \mathrm{m}$.

bryos injected with MOs made to block $p 27^{X i c 1}$ translation were not examined at later stages of development to determine whether neural cells eventually exited the cell cycle (Carruthers et al., 2003; Vernon et al., 2003). To learn whether zebrafish embryos that lack $c d k n 1 c$ function undergo a delayed primary neurogenesis, we examined $\mathrm{MO}$-injected wild-type embryos at 20 hpf using RNA probe to detect isl 2 expression, which marks Rohon-Beard ( $\mathrm{RB})$ sensory neurons and $\mathrm{CaP}$ and $\mathrm{VaP}$ motor neurons (Appel et al., 1995). Both $\mathrm{RB}$ and $\mathrm{CaP}$ and VaP neurons were evident at $20 \mathrm{hpf}$ ( $n=128$ embryos) (Fig. $3 F$ ), indicating that loss of $c d k n 1 c$ function delayed but did not prevent primary neurogenesis. Notably, $c d k n 1 c$ MO-injected embryos had a small but statistically significant increase in the number of $i s l 2^{+}$primary motor neurons. Whereas each ventral hemisegment of control embryos had one $(\mathrm{CaP})$ or two $(\mathrm{CaP}$ and $\mathrm{VaP})$ isl $2^{+}$cells (Fig. $3 E$ ), $36.7 \%$ of the $c d k n 1 c$ MO-injected embryos had one to three

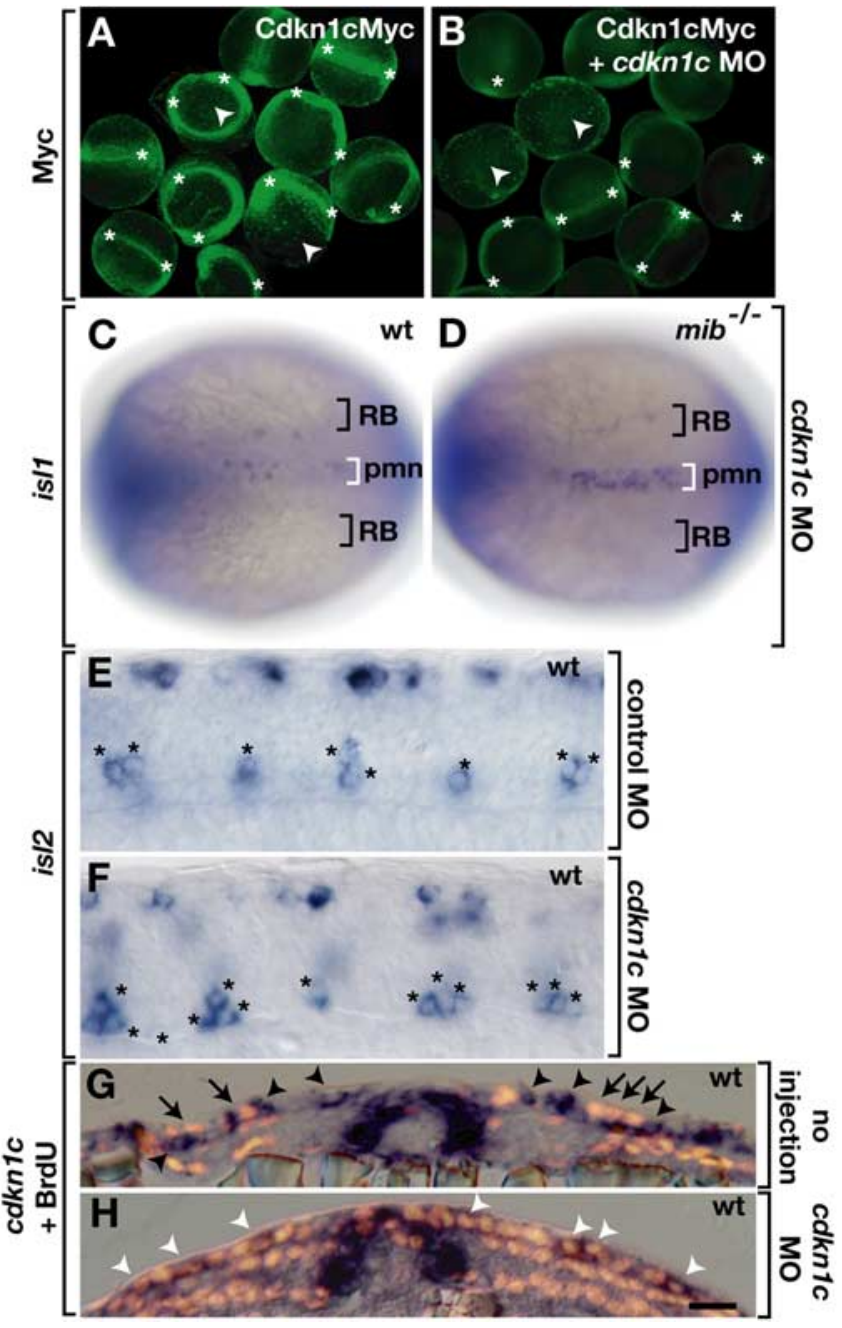

Figure 3. $\quad c d k n 1 c$ function is required for timely production of primary neurons in wild-type and mib ${ }^{-/-}$embryos. $\boldsymbol{A}, \boldsymbol{B}, \mathrm{Tg}$ (hsp70:cdkn1c-myc) (11 hpf) embryos heat shocked at $7.5 \mathrm{hpf}$ and processed for anti-Myc immunocytochemistry (green fluorescence). $\boldsymbol{A}$, Control embryos had high levels of fluorescence in the embryonic axis (asterisks) and some expression in yolk (small spots indicated by arrowheads). B, Embryos injected with cdkn1c MO had low levels of fluorescence in the embryonic axis, although some fluorescence was evident in yolk. $C, D$, Dorsal views, $10.5 \mathrm{hpf}$ embryos, anterior to left. The white brackets mark primary motor neuron (pmn) domain of medial neural plate. The black brackets mark RB sensory neuron domains of lateral neural plate. C, Wild-type embryo injected with cdkn 1 c MO produced few is $/ 1^{+}$neurons in the pmn or RB domains. $\boldsymbol{D}, \mathrm{mib}^{-1-}$ embryo injected with $\mathrm{cdkn} 1 \mathrm{c} \mathrm{MO}$. The dense cluster of is $11^{+}$ neurons in the pmn domain reveals that this was a mutant embryo, but, overall, there was a deficit of primary neurons. $\boldsymbol{E}, \boldsymbol{F}$, Side views of whole-mount embryos, focused on trunk spinal cord. Dorsal is up, and anterior to the left. $\boldsymbol{E}$, Wild-type control embryo hybridized with is/2 probe to reveal one or two primary motor neurons in ventral spinal cord (asterisks) and RohonBeard sensory neurons in dorsal spinal cord. $F$, Wild-type embryo injected with cdkn 1c M0. One to three is $/ 2^{+}$cells per hemisegment formed in ventral spinal cord (asterisks). $\mathbf{G}, \boldsymbol{H}$, Transverse sections of $11 \mathrm{hpf}$ embryos processed for cdkn1c in situ RNA hybridization (blue) and BrdU immunocytochemistry (pink). G, cdkn1c- (arrowheads) and BrdU- (arrows) labeled different neural plate cells in control embryo. $\boldsymbol{H}$, cdkn1c-positive cells incorporated BrdU (white arrowheads) in embryo injected with cdkn1c MO. Scale bar: (in $\boldsymbol{H}) \boldsymbol{A}, \boldsymbol{B}, 400 \mu \mathrm{m} ; \boldsymbol{C}, \boldsymbol{D}, 100 \mu \mathrm{m} ; \boldsymbol{E}-\boldsymbol{H}$, $25 \mu \mathrm{m}$. wt, Wild type.

is $2^{+}$cells per hemisegment (Fig. 3F). Cells counted from 12 hemisegments each of five control and five $c d k n 1 c$ MO-injected embryos with excess primary motor neurons revealed an average of 1.5 and 2.4 isl $2^{+}$cells, respectively $(p<0.001)$. To test the possibility that excess primary motor neurons resulted from excess cell proliferation, we incubated embryos with the thymidine analog BrdU to label S-phase cells. In wild-type embryos, neural 

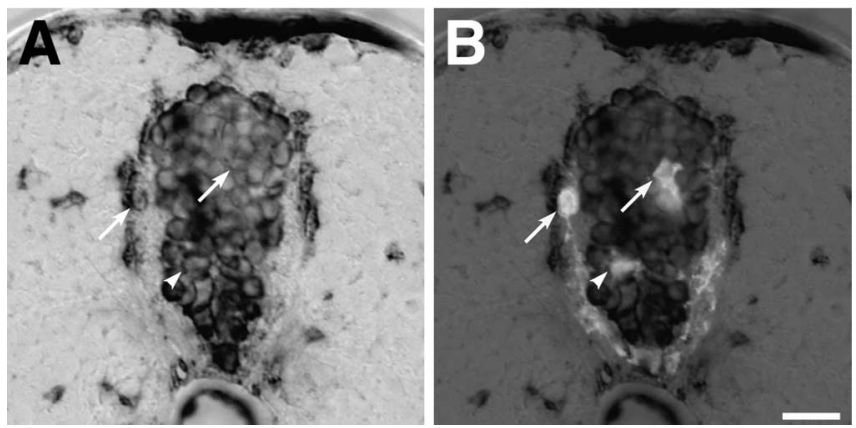

Figure 4. OPCs express cdkn1c. Transverse sections of $3 \mathrm{dpf} \mathrm{Tg}$ (olig2:egfp) embryo hybridized with $c d k n 1 c$ RNA probe. Differential interference contrast (DIC; $\boldsymbol{A})$ and combined DIC and fluorescence images of same section $(\boldsymbol{B})$ are shown. The arrows indicate $c d k n 1 c^{+} \mathrm{OPCs}$. The arrowhead marks an $\mathrm{EGFP}^{+}, \mathrm{cdkn} 1 c^{+}$cell at or near the oligodendrocyte origin in ventral spinal cord. Scale bar: (in $\boldsymbol{B}) 20 \mu \mathrm{m}$.

plate cells that expressed $c d k n 1 c$ did not incorporate BrdU (Fig. $3 G$ ). In contrast, many neural plate cells of embryos injected with $c d k n 1 c \mathrm{MO}$ were both $c d k n 1 c$ positive and BrdU positive (Fig. $3 H$ ). These data indicate that $c d k n 1 c$ function is necessary for some neural plate cells to stop dividing and differentiate as primary motor neurons on schedule.

\section{$c d k n 1 c$ function is required for oligodendrocyte specification in zebrafish}

The above data are consistent with the following model. During early stages of neural development, cells that have low levels of Notch activity express $c d k n 1 c$, exit the cell cycle, and develop as primary neurons, whereas those that have high levels of Notch activity do not express $c d k n 1 c$ and remain proliferative. Subsequently, some of these precursors escape from Notch inhibition, express $c d k n 1 c$, and develop as secondary neurons. This model raises the question as to whether, after the period of neuron production, $c d k n 1 c$ function is necessary for glial cell specification. To investigate a possible role in oligodendrocyte specification, we first examined $c d k n 1 c$ expression by in situ RNA hybridization to transverse sections obtained from $\mathrm{Tg}$ (oligz:egfp) embryos (Shin et al., 2003; Park et al., 2004). At 3 dpf, many spinal cord cells expressed $c d k n 1 c$, including olig2: $\mathrm{EGFP}^{+} \mathrm{OPCs}$ and differentiating oligodendrocytes (Fig. 4A,B). Second, we injected $c d k n 1 c$ MO into $\operatorname{Tg}$ (olig2:egfp) embryos. At $3 \mathrm{dpf}$, when dorsally migrated OPCs were evident in control embryos, similarly staged $c d k n 1 c$ MO-injected embryos had few dorsal OPCs (data not shown) (Fig. 5H). OPCs were still absent from dorsal spinal cord at $4 \mathrm{dpf}$ (Fig. $5 \mathrm{~B}$ ) and $5 \mathrm{dpf}$ (data not shown), suggesting that OPCs were not simply delayed in their development. Because motor neuron expression of EGFP might obscure OPCs that failed to migrate dorsally in $c d k n 1 c$ MO-injected embryos, we tested $p l p / d m 20$ expression, which marks the myelinating subpopulation of oligodendrocyte lineage cells (Brosamle and Halpern, 2002; Park et al., 2002). Whereas 4 dpf wild-type embryos had numerous $p l p / d m 20^{+}$cells in dorsal and ventral spinal cord (Fig. 5C), 83 of 96 similarly staged $c d k n 1 c$ MO-injected embryos had very few $p l p / d m 20^{+}$spinal cord cells (Fig. 5D). Additionally, we used immunocytochemistry to examine expression of Sox10, which is the earliest known marker of OPCs in zebrafish (Park et al., 2002). Relative to wild type (Fig. 5E), $c d k n 1 c$ MO-injected embryos had few Sox $10^{+}$OPCs (Fig. $5 F$ ).

Notch-signaling-deficient embryos also have excess primary neurons and a deficit of oligodendrocytes (Appel et al., 2001; Park and Appel, 2003). In these embryos, all spinal cord precursors

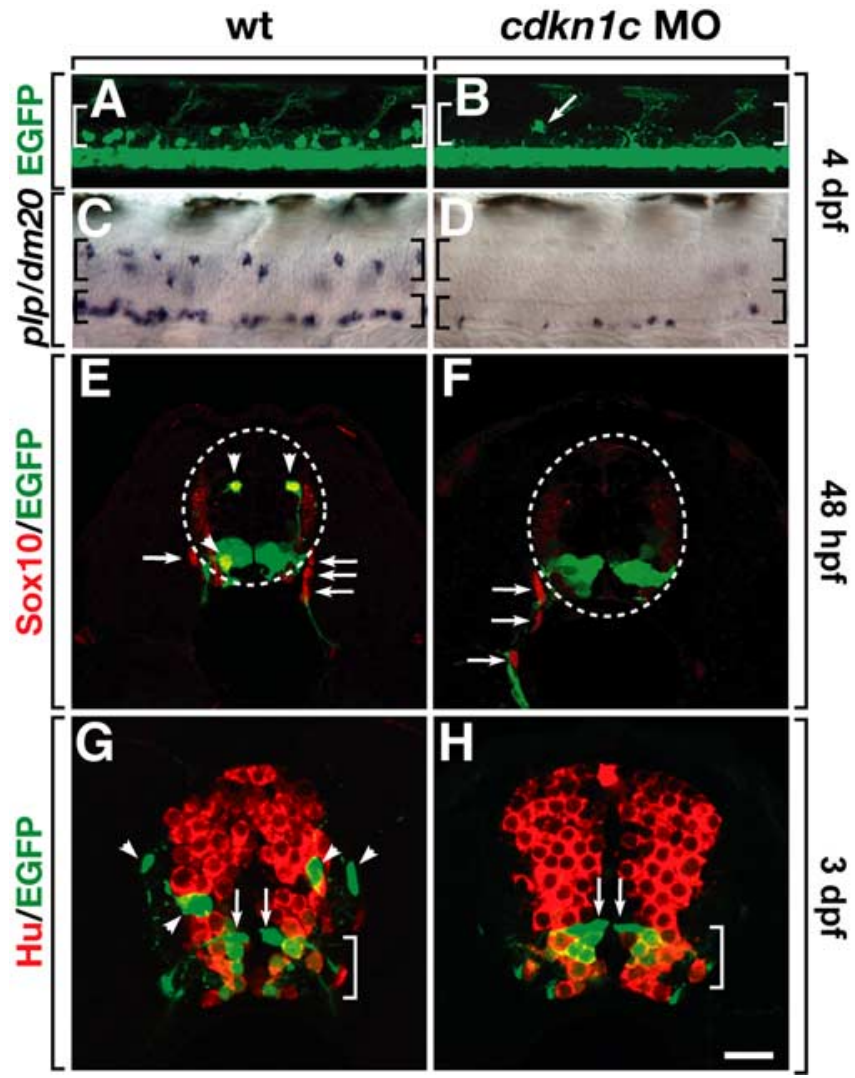

Figure 5. $\quad c d k n 1 c$ function is necessary for $\mathrm{OPC}$ specification. $A-D$, Side views of wholemount $4 \mathrm{dpf}$ embryos, dorsal up and anterior to the left. $\boldsymbol{A}$, Control Tg(oligz:egfp) embryo showing numerous olig2:EGFP ${ }^{+}$OPCs in dorsal spinal cord (brackets). $B, A$ cdkn1c M0-injected Tg(olig2:egfp) embryo. A single OPC (arrow) is evident in dorsal spinal cord (brackets). C, Control embryo hybridized with $p / p / d m 20$ RNA probe. Numerous $p / p / d m 20^{+}$oligodendrocytes occupy dorsal and ventral spinal cord (top and bottom brackets, respectively). $\boldsymbol{D}$, Embryo injected with cdkn1c M0. Only a few oligodendrocytes are evident in ventral spinal cord (bottom bracket), and none are present in dorsal spinal cord (top bracket). $\boldsymbol{E}-\boldsymbol{H}$, Transverse sections of Tg(oligz:egfp) embryos, dorsal up. $\boldsymbol{E}, \boldsymbol{F}$, Combined anti-Sox10 (red fluorescence) and olig2:EGFP labeling of 48 hpf embryos. Arrows mark Sox $10^{+}$Schwann cells, and dashed circle outlines spinal cord. $\boldsymbol{E}$, Arrowheads indicate EGFP ${ }^{+}$, Sox $10^{+} \mathrm{OPC}$ in spinal cord of control embryo. $F$, Embryo injected with cdkn1c M0 expressed EGFP in ventral spinal cord and Sox10 outside the spinal cord normally but produced no OPCs. G, $\boldsymbol{H}$, Tg(oligz:egfp) embryos labeled with anti-Hu antibody to mark neurons (red). $G$, Control embryo had EGFP ${ }^{+}, \mathrm{Hu}^{-} \mathrm{OPCs}$ (arrowheads), EGFP ${ }^{+}, \mathrm{Hu}$ precursors located near the ventral ventricle (arrows), and EGFP ${ }^{+}, \mathrm{Hu}^{+}$ventral neurons (bracket). $\boldsymbol{H}$, cdkn1c M0-injected embryo had EGFP ${ }^{+}, \mathrm{Hu}^{-}$precursors (arrows) and EGFP ${ }^{+}$ $\mathrm{Hu}^{+}$ventral neurons (bracket) but no OPCs. Scale bar: (in $\left.\boldsymbol{H}\right) \boldsymbol{A}-\boldsymbol{D}, 40 \mu \mathrm{m} ; \boldsymbol{E}-\boldsymbol{F}, 20 \mu \mathrm{m}$; and $\boldsymbol{G}$, H, $15 \mu \mathrm{m}$. wt, Wild type.

stop dividing too soon and differentiate as neurons, and this is revealed as an excess of $\mathrm{Hu}^{+}$neurons and absence of $\mathrm{Hu}^{-}$cells at $1 \mathrm{dpf}$. To determine whether $c d k n 1 c$-deficient embryos similarly lack oligodendrocytes because they fail to maintain the precursors that give rise to them, we labeled $c d k n 1 c$ MO-injected $\mathrm{Tg}$ (olig2:egfp) embryos with anti-Hu antibody. At $3 \mathrm{dpf}$, wildtype embryos had dorsal and ventral $\mathrm{EGFP}^{+}, \mathrm{Hu}^{-}$OPCs, ventral $\mathrm{EGFP}^{+}, \mathrm{Hu}^{+}$neurons, and $\mathrm{EGFP}^{+}, \mathrm{Hu}^{-}$cells near the ventral ventricle (Fig. 5G), which we showed previously are proliferative (Park et al., 2004). Embryos injected with $c d k n 1 c$ MO had $\mathrm{EGFP}^{+}, \mathrm{Hu}^{+}$neurons but, as shown above, no OPCs (Fig. $5 H$ ). In contrast to Notch-signaling-deficient embryos, $c d k n 1 c \mathrm{MO}$ injected embryos maintained ventral $\mathrm{EGFP}^{+}, \mathrm{Hu}^{-}$precursor cells, and the number and distribution of neurons appeared normal (Fig. $5 H$ ). Taken together, these data indicate that $c d k n 1 c$ 


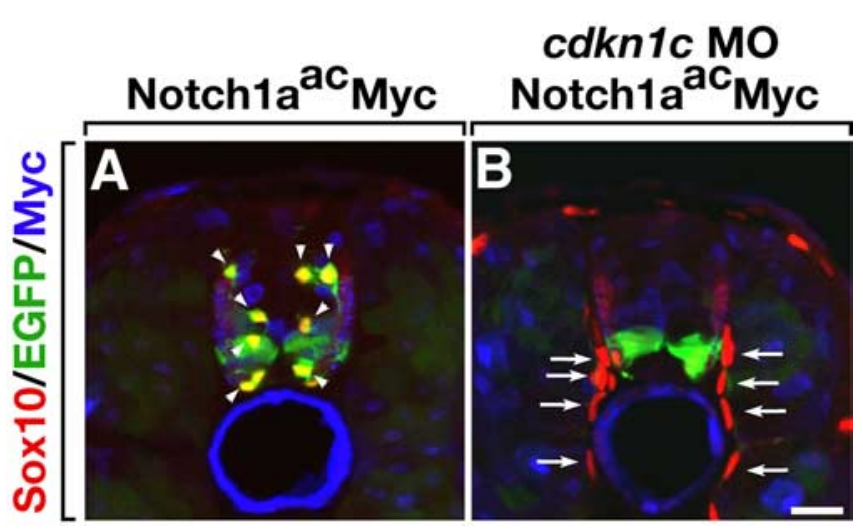

Figure 6. Formation of excess OPCs by Notch activity is dependent on cdkn1c function. Panels show transverse sections, dorsal up, of $T g$ (hsp70:GAL4); $T g$ (UAS:Notch1a ${ }^{a c}$-myc); Tg(olig2:egfp) embryos. $\boldsymbol{A}$, Control embryo in which heat shock at 24 hpf produced excess $\mathrm{EGFP}^{+}$, Sox $10^{+}$OPCs (arrowheads). B, Embryo injected with cdkn $1 \mathrm{c} \mathrm{M0}$ and heat shocked at $24 \mathrm{hpf}$ lacked OPCs. Anti-Myc labeling (blue) reveals Notch1 a ${ }^{\mathrm{a}}$ Myc expression that persisted after heat-shock induction. The arrows mark Sox $10^{+}$Schwann cells. Schwann cells are distributed intermittently along the anteroposterior axis, which accounts for their absence in the transverse section shown in $\boldsymbol{A}$. Scale bar: (in $\boldsymbol{B}) 20 \mu \mathrm{m}$.

function is necessary to specify oligodendrocyte lineage cells from olig2: $\mathrm{EGFP}^{+}$spinal cord precursors.

Previously, we showed that embryos in which a constitutively active form of Notchla was expressed beginning at $24 \mathrm{hpf}$ had about a twofold excess of OPCs (Park and Appel, 2003). To determine whether formation of excess OPCs is dependent on $c d k n 1 c$ function, we injected $c d k n 1 c$ MO into cleavage stage $\operatorname{Tg}$ (hsp70:GAL4); $\operatorname{Tg}$ (UAS:Notch1a ${ }^{a c}$-myc); $T g($ olig2:egfp) embryos and induced expression of constitutively active Notch by heat shock at $24 \mathrm{hpf}$. At $48 \mathrm{hpf}$, noninjected embryos had excess Sox $10^{+}$, olig2:EGFP ${ }^{+}$OPCs (Fig. 6 A), consistent with our previous results. In contrast, embryos injected with $c d k n 1 c \mathrm{MO}$ had a deficit of OPCs (Fig. $6 B$ ), showing that $c d k n 1 c$ function is required to mediate OPC specification by Notch signaling.

Our data are paradoxical, in that we showed that Notch inhibits $c d k n 1 c$ expression and can promote formation of excess OPCs but that $c d k n 1 c$ function is required for OPC specification. We speculated that ventral neural precursors that begin to escape Notch inhibition, even after transiently induced expression of constitutively active Notch, and express low levels of $c d k n 1 c$ after neurons are produced are specified as OPCs. One test of this idea would be to block Cdkn1c function just before OPC specification. However, we lack a method to do this. Instead, we heat shocked $\operatorname{Tg}(h s p 70: c d k n 1 c-m y c)$ embryos to induce Cdkn1cMyc expression at different times. We first tested the effect of $c d k n 1 c$ overexpression by heat shocking $\operatorname{Tg}(h s p 70: c d k n 1 c-m y c)$ embryos at $24 \mathrm{hpf}$. This induced expression of CdknlcMyc at high level but did not cause premature formation of OPCs at $32 \mathrm{hpf}$ (Fig. $7 A, B)$. Next, we induced Cdkn1cMyc expression by heat shock at $28 \mathrm{hpf}$ and analyzed embryos at $48 \mathrm{hpf}, \sim 12 \mathrm{~h}$ after Sox $10^{+}$OPCs are normally first specified. The number and distribution of OPCs was similar to wild type (compare Figs. $7 C, D, 5 E$ ), indicating that Cdknlc overexpression is not sufficient to promote formation of excess or ectopic OPCs at their normal time of development. Finally, we asked whether time-dependent expression of Cdkn1cMyc could rescue the OPC deficit caused by $c d k n 1 c$ MO injection. Although we showed above that $c d k n 1 c \mathrm{MO}$ can interfere with Cdkn1Myc expression encoded by a transgene during early development, we reasoned that sufficiently high transgene expression should produce transcripts in excess of $\mathrm{MO}$ as the $\mathrm{MO}$

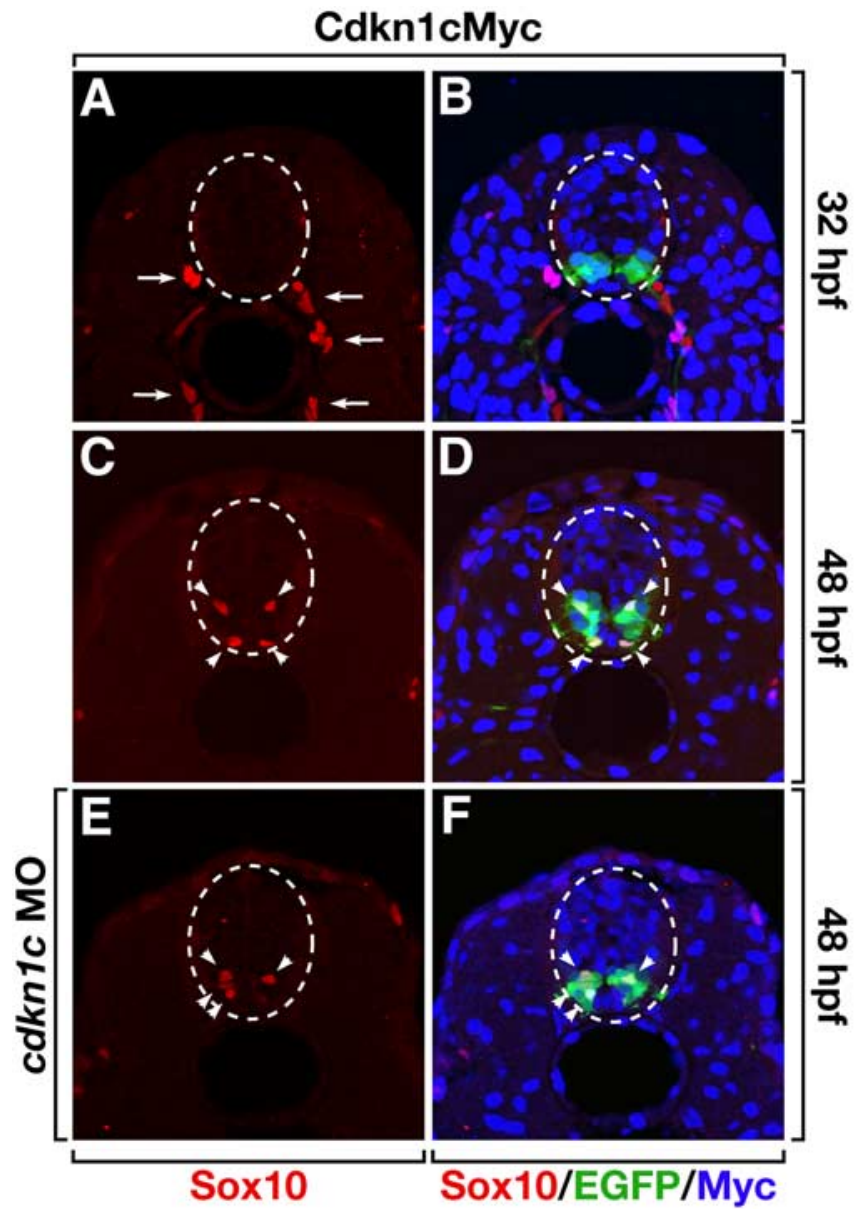

Figure 7. Time-dependent expression of $c d k n 1 c$ rescues the OPC deficit produced by $c d k n 1 c$ M0. All images are of transverse sections of $T g$ (hsp70:cdkn1c-myc); $T g$ (oligz:egfp) embryos, dorsal up. The dashed circle outlines the spinal cord, and the arrows mark Sox $10^{+}$Schwann cells. $A, B$, Induction of Cdkn1cMyc expression at $24 \mathrm{hpf}$ did not promote premature formation of Sox $10^{+}$, olig2:EGFP ${ }^{+}$OPCs at $32 \mathrm{hpf}$. C, $\boldsymbol{D}$, Induction of Cdkn1cMyc expression at $28 \mathrm{hpf}$ did not promote formation of excess or ectopic Sox $10^{+}{ }^{+}$, olig2:EGFP ${ }^{+}$OPCs at $48 \mathrm{hpf}$. $E$, $F$, Induction of Cdkn1cMyc expression at $28 \mathrm{hpf}$ produced OPCs (arrowheads) in cdkn1c M0-injected embryo at $48 \mathrm{hpf}$.

becomes depleted at later stages of development. Indeed, antiMyc immunocytochemistry revealed substantial Cdkn1cMyc expression when transgenic embryos were heat shocked at $28 \mathrm{hpf}$ (Fig. $7 F$ ). These embryos produced, on average, 2.30 OPCs per section (66 sections from eight embryos) (Fig. 7E), in contrast to nontransgenic, $c d k n 1 c \mathrm{MO}$-injected embryos, which produced an average of 0.86 OPCs per section (66 sections from eight embryos; $p<0.00001$ ) (images not shown but compare with Fig. $5 B, D, F, H)$. Thus, $c d k n 1 c$ expression induced after most neurons are produced but before oligodendrocyte specification rescues the oligodendrocyte deficit caused by $c d k n 1 c \mathrm{MO}$. We conclude that specification of oligodendrocyte fate from olig $2^{+}$spinal cord precursors requires $c d k n 1 c$ function.

\section{Discussion}

Notch signaling coordinates neurogenesis by regulating cdkn1c expression

One critical mechanism for coordinating neural cell proliferation, specification, and differentiation is Notch signaling. Typically, membrane-bound ligands, such as Delta proteins, interact with transmembrane Notch receptors, causing transduction of a signal to the nucleus of Notch-expressing cells that influences 
gene transcription (Kadesch, 2004). Among the consequences of Delta-Notch signaling activity in the CNS is differential expression of proneural class genes, such as neurogenin1, which encode basic helix-loop-helix (bHLH) transcription factors that promote cell-cycle exit and neuronal development (Kageyama and Ohtsuka, 1999). Cells that have relatively high Notch activity inhibit proneural gene expression and remain in the cell cycle, whereas those with low Notch activity express proneural genes, stop dividing, and develop as neurons. Functional tests of the Notch pathway reveal that signaling is important to diversify neural cell fate in vertebrate embryos. For example, zebrafish embryos deficient for Notch activity failed to maintain proliferative precursors and produced excess early born neurons at the expense of later-forming neurons and glia (Appel and Eisen, 1998; Appel et al., 2001; Park and Appel, 2003), and expression of constitutively active Notch blocked neuronal development and routed precursors into glial developmental pathways (Furukawa et al., 2000; Scheer et al., 2001; Park and Appel, 2003). Evidence also points to a role for Notch activity in regulating development of oligodendrocyte lineage cells. In culture, Notch activity inhibited differentiation of OPCs (Wang et al., 1998).

Previously published data raised the possibility that genes regulated by Notch activity might include those that encode Cdkis. For example, flies deficient for the Cdki Decapo underwent additional rounds of cell division, whereas overexpression of Decapo caused cells to stop dividing prematurely (Lane et al., 1996). Similarly, mice and frogs that lacked $\mathrm{p} 27^{\mathrm{Kip} 1}$ and $\mathrm{p} 27^{\mathrm{Xicl}}$ function, respectively, had increased numbers of proliferating cells (Kiyokawa et al., 1996; Carruthers et al., 2003; Vernon et al., 2003), and overexpression of $\mathrm{p} 27^{\mathrm{Xicl}}$ in frog embryos reduced proliferation and caused neural plate cells to form excess primary neurons (Hardcastle and Papalopulu, 2000; Vernon et al., 2003). Thus, Notch activity could prevent cell-cycle exit and neuronal differentiation by inhibiting $c d k i$ gene expression. In principle, this could occur because Notch activity directly inhibits $c d k i$ gene transcription or Notch activity inhibits expression of genes that encode activators of $c d k i$ transcription. Consistent with the latter possibility, P19 embryonal carcinoma cells transfected with constructs that expressed proneural factors expressed elevated levels of $\mathrm{p} 27^{\mathrm{kip} 1}$ and withdrew from the cell cycle (Farah et al., 2000). Genomic DNA near the $p 21^{\text {Cip } 1}$ transcription start site contains E-box sequences, which bind bHLH transcription factors, and these mediate enhancement of $p 21^{\text {Cip } 1}$ transcription by bHLH proteins in cell-culture assays (Pagliuca et al., 2000). In flies, decapo expression is positively regulated by asense, which encodes a proneural bHLH factor (Wallace et al., 2000).

Our data establish that Notch signaling regulates $c d k n 1 c$ expression within the CNS of zebrafish embryos. Nonproliferative neural plate cells expressed $c d k n 1 c$ in a mosaic pattern consistent with the pattern of primary neuron formation. Excess neural plate cells expressed $c d k n 1 c$ and developed as primary neurons in mutant embryos that were deficient for Notch signaling, whereas embryos that expressed a constitutively active Notch had few $c d k n 1 c^{+}$neural plate cells and deficits of primary neurons. At late stages of neurogenesis a conditional block of Notch signaling activity also caused excess spinal cord cells to express $c d k n 1 c$, stop proliferating, and develop as neurons. Thus, Notch signaling may regulate the orderly formation of neurons over time by regulating transcription of $c d k n 1 c$.

\section{$c d k n 1 c$ function is required for timely formation of neurons} Previous work using frogs showed that $p 27^{X i c 1}$ function is required to limit neural cell proliferation and promote formation of primary neurons from neural plate cells (Carruthers et al., 2003; Vernon et al., 2003). We found a similar effect for loss of $c d k n 1 c$ function in zebrafish embryos. However, we also learned that these embryos eventually produced primary motor neurons, sometimes in excess number. This result is remarkably similar to the consequences of disrupted Cdki activity in nematodes, wherein vulva precursor cells underwent only one additional round of cell division to produce excess ventral hypodermal cells in the absence of cki-1function (Hong et al., 1998). Thus, the simplest interpretation of our data is that, in the absence of $c d k n 1 c$ activity, neural plate cells proceed through one extra division but remain competent to respond to signals that specify primary motor neuron fate.

This interpretation has an interesting implication. We have analyzed clones made by marking single cells within a precursor domain that gives rise to motor neurons, interneurons, and oligodendrocytes (Park et al., 2004). The size and cell-type composition of the clones was highly variable, suggesting that specification of cell fate results from integration of extrinsic signals that change with time. Consistent with this, we found that timedependent manipulation of Notch and Hedgehog signaling alters the number of cells specified for motor neuron, interneuron, and oligodendrocyte fates (Park and Appel, 2003; Park et al., 2004). If an obligate relationship exists between neural cell-cycle exit and fate specification, we would predict that, in the absence of $c d k n 1 c$ function, medial neural plate precursors that normally give rise to primary motor neurons would instead produce cells that develop after primary motor neurons. Because this, apparently, is not the case, our results raise the possibility that precursors can be specified for primary motor neuron fate and retain this identity through an additional round of cell division. Thus, $c d k n 1 c$ function probably is only a part of a mechanism that coordinates neural cell cycle exit and neuronal specification.

\section{$c d k n 1 c$ function is required for oligodendrocyte specification} Cdkis also play important roles in regulating proliferation and differentiation of OPCs, proliferative cells committed to the oligodendrocyte lineage. For example, relatively undifferentiated OPCs expressed low levels of $\mathrm{p} 27^{\mathrm{kip} 1}$ protein, whereas progressively more fully differentiated cells expressed higher levels (Durand et al., 1997). Cultured OPCs derived from $p 27^{k i p 1}$ mutant mice proliferated more than OPCs from wild-type mice (Casaccia-Bonnefil et al., 1997; Durand et al., 1998; Zezula et al., 2001) and had impaired ability to develop the multiple branching processes characteristic of differentiated oligodendrocytes (Casaccia-Bonnefil et al., 1997; Zezula et al., 2001). p27 ${ }^{k i p 1} \mathrm{mu}-$ tant mice had more proliferative OPCs than wild type but formed myelinating oligodendrocytes on schedule (Casaccia-Bonnefil et al., 1999). In contrast, although OPCs from $p 21^{\text {Cip } 1}$ mutant mice had the same proliferative characteristics as wild-type OPCs, they did not fully differentiate (Zezula et al., 2001). One caveat of the mouse loss-of-function tests is that mice express multiple $c d k i$ genes in overlapping patterns within the nervous system (Zhu and Skoultchi, 2001). Thus, mouse Cdkis might have significant functional redundancy. In contrast to the mouse $p 21$ and $p 27$ mutants, in which OPCs are formed but do not proliferate or differentiate properly, we found that zebrafish embryos that had reduced function of $c d k n 1 c$, a $p 57$ homolog, did not produce spinal cord OPCs. Instead, the olig2:EGFP ${ }^{+}$precursors that normally give rise to OPCs appeared to remain in an undifferentiated state. Spinal cord cells of zebrafish embryos express high levels of $c d k n 1 c$ but not $c d k n 1 b$, a $p 27$ homolog, and we did not find a $p 21$ homolog. This raises the possibility that, in zebrafish, a single 
gene provides most or all of Cdki function necessary to regulate oligodendrocyte development. Thus, the absence of spinal cord OPCs in $c d k n 1 c$-deficient zebrafish embryos reveals a new role for Cdki function in specification of OPCs from proliferative neural precursors. In frogs, retinal cells lipofected with antisense $p 27^{X i c 1}$ RNA formed fewer Müller glia than controls (Ohnuma et al., 1999). These data raise the possibility that Cdkis have widespread roles in glial specification.

We also found that expression of $c d k n 1 c$ from a transgene before OPC specification restored OPC development in embryos injected with $c d k n 1 c \mathrm{MO}$. This provides strong evidence for our conclusion that $c d k n 1 c$ function is necessary for OPC specification. However, transgenic $c d k n 1 c$ expression was not sufficient to induce formation of OPCs prematurely or in excess number at the normal time of OPC specification. This is in contrast to overexpression of $\mathrm{p} 27^{\mathrm{Xicl}}$ in frog retina, which promoted formation of excess Müller glia and a concomitant reduction in the number of bipolar neurons (Ohnuma et al., 1999). Previously, we showed that oligodendrocyte specification is temporally and spatially constrained (Park and Appel, 2003; Park et al., 2004). Thus, $c d k n 1 c$ does not function as a determination factor but as a necessary trigger for cells that are competent to respond to other signals that instruct oligodendrocyte specification.

Rising Cdki protein levels may regulate OPC cell-cycle exit and differentiation. We showed that Notch inhibition of $c d k n 1 c$ expression limits formation of neurons and maintains a population of neural precursors, yet that $c d k n 1 c$ function is also necessary for precursors to give rise to OPCs. We propose that, after formation of most neurons, remaining ventral spinal cord precursors that escape Notch-mediated inhibition begin to express $c d k n 1 c$ and that the initial low level of Cdkn1c protein triggers OPC specification without causing cells to stop dividing. Subsequent accumulation of Cdkn proteins might then drive OPCs from the cell cycle and promote oligodendrocyte differentiation.

\section{References}

Altschul SF, Gish W, Miller W, Myers EW, Lipman DJ (1990) Basic local alignment search tool. J Mol Biol 215:403-410.

Appel B, Eisen JS (1998) Regulation of neuronal specification in the zebrafish spinal cord by Delta function. Development 125:371-380.

Appel B, Korzh V, Glasgow E, Thor S, Edlund T, Dawid IB, Eisen JS (1995) Motoneuron fate specification revealed by patterned LIM homeobox gene expression in embryonic zebrafish. Development 121:4117-4125.

Appel B, Fritz A, Westerfield M, Grunwald DJ, Eisen JS, Riley BB (1999) Delta-mediated specification of midline cell fates in zebrafish embryos. Curr Biol 9:247-256.

Appel B, Givan LA, Eisen JS (2001) Delta-Notch signaling and lateral inhibition in zebrafish spinal cord development. BMC Dev Biol 1:13.

Baumann N, Pham-Dinh D (2001) Biology of oligodendrocyte and myelin in the mammalian central nervous system. Physiol Rev 81:871-927.

Brosamle C, Halpern ME (2002) Characterization of myelination in the developing zebrafish. Glia 39:47-57.

Carruthers S, Mason J, Papalopulu N (2003) Depletion of the cell-cycle inhibitor p27(Xicl) impairs neuronal differentiation and increases the number of $\mathrm{ElrC}(+)$ progenitor cells in Xenopus tropicalis. Mech Dev 120:607-616.

Casaccia-Bonnefil P, Tikoo R, Kiyokawa H, Friedrich Jr V, Chao MV, Koff A (1997) Oligodendrocyte precursor differentiation is perturbed in the absence of the cyclin-dependent kinase inhibitor p27Kip1. Genes Dev 11:2335-2346.

Casaccia-Bonnefil P, Hardy RJ, Teng KK, Levine JM, Koff A, Chao MV (1999) Loss of p27Kip1 function results in increased proliferative capacity of oligodendrocyte progenitors but unaltered timing of differentiation. Development 126:4027-4037.

Cunningham JJ, Roussel MF (2001) Cyclin-dependent kinase inhibitors in the development of the central nervous system. Cell Growth Differ 12:387-396. de Nooij JC, Letendre MA, Hariharan IK (1996) A cyclin-dependent kinase inhibitor, Dacapo, is necessary for timely exit from the cell cycle during Drosophila embryogenesis. Cell 87:1237-1247.

Durand B, Gao FB, Raff M (1997) Accumulation of the cyclin-dependent kinase inhibitor p27/Kip1 and the timing of oligodendrocyte differentiation. EMBO J 16:306-317.

Durand B, Fero ML, Roberts JM, Raff MC (1998) p27Kip1 alters the response of cells to mitogen and is part of a cell-intrinsic timer that arrests the cell cycle and initiates differentiation. Curr Biol 8:431-440.

Farah MH, Olson JM, Sucic HB, Hume RI, Tapscott SJ, Turner DL (2000) Generation of neurons by transient expression of neural bHLH proteins in mammalian cells. Development 127:693-702.

Fukuyama M, Gendreau SB, Derry WB, Rothman JH (2003) Essential embryonic roles of the CKI-1 cyclin-dependent kinase inhibitor in cell-cycle exit and morphogenesis in C. elegans. Dev Biol 260:273-286.

Furukawa T, Mukherjee S, Bao ZZ, Morrow EM, Cepko CL (2000) rax, Hes1, and notch1 promote the formation of Muller glia by postnatal retinal progenitor cells. Neuron 26:383-394.

Hardcastle Z, Papalopulu N (2000) Distinct effects of XBF-1 in regulating the cell cycle inhibitor p27(XIC1) and imparting a neural fate. Development 127:1303-1314.

Hauptmann G, Gerster T (2000) Multicolor whole-mount in situ hybridization. Methods Mol Biol 137:139-148.

Holley SA, Geisler R, Nusslein-Volhard C (2000) Control of herl expression during zebrafish somitogenesis by a delta-dependent oscillator and an independent wave-front activity. Genes Dev 14:1678-1690.

Hong Y, Roy R, Ambros V (1998) Developmental regulation of a cyclindependent kinase inhibitor controls postembryonic cell cycle progression in Caenorhabditis elegans. Development 125:3585-3597.

Horner PJ, Power AE, Kempermann G, Kuhn HG, Palmer TD, Winkler J, Thal LJ, Gage FH (2000) Proliferation and differentiation of progenitor cells throughout the intact adult rat spinal cord. J Neurosci 20:2218-2228.

Itoh M, Kim CH, Palardy G, Oda T, Jiang YJ, Maust D, Yeo SY, Lorick K, Wright GJ, Ariza-McNaughton L, Weissman AM, Lewis J, Chandrasekharappa SC, Chitnis AB (2003) Mind bomb is a ubiquitin ligase that is essential for efficient activation of Notch signaling by Delta. Dev Cell 4:67-82.

Jiang YJ, Brand M, Heisenberg CP, Beuchle D, Furutani-Seiki M, Kelsh RN, Warga RM, Granato M, Haffter P, Hammerschmidt M, Kane DA, Mullins MC, Odenthal J, van Eeden FJ, Nusslein-Volhard C (1996) Mutations affecting neurogenesis and brain morphology in the zebrafish, Danio rerio. Development 123:205-216.

Kadesch T (2004) Notch signaling: the demise of elegant simplicity. Curr Opin Genet Dev 14:506-512.

Kageyama R, Ohtsuka T (1999) The Notch-Hes pathway in mammalian neural development. Cell Res 9:179-188.

Kimmel CB, Ballard WW, Kimmel SR, Ullmann B, Schilling TF (1995) Stages of embryonic development of the zebrafish. Dev Dyn 203:253-310.

Kiyokawa H, Kineman RD, Manova-Todorova KO, Soares VC, Hoffman ES, Ono M, Khanam D, Hayday AC, Frohman LA, Koff A (1996) Enhanced growth of mice lacking the cyclin-dependent kinase inhibitor function of p27(Kip1). Cell 85:721-732.

Lane ME, Sauer K, Wallace K, Jan YN, Lehner CF, Vaessin H (1996) Dacapo, a cyclin-dependent kinase inhibitor, stops cell proliferation during Drosophila development. Cell 87:1225-1235.

Latimer AJ, Shin J, Appel B (2005) her9 promotes floor plate development in zebrafish. Dev Dyn 232:1098-1104.

Levine EM, Close J, Fero M, Ostrovsky A, Reh TA (2000) p27(Kip1) regulates cell cycle withdrawal of late multipotent progenitor cells in the mammalian retina. Dev Biol 219:299-314.

Levison SW, Young GM, Goldman JE (1999) Cycling cells in the adult rat neocortex preferentially generate oligodendroglia. J Neurosci Res 57:435-446.

Lowenheim H, Furness DN, Kil J, Zinn C, Gultig K, Fero ML, Frost D, Gummer AW, Roberts JM, Rubel EW, Hackney CM, Zenner HP (1999) Gene disruption of $\mathrm{p} 27(\mathrm{Kip} 1)$ allows cell proliferation in the postnatal and adult organ of Corti. Proc Natl Acad Sci USA 96:4084-4088.

McConnell SK, Kaznowski CE (1991) Cell cycle dependence of laminar determination in developing neocortex. Science 254:282-285.

Miller RH (2002) Regulation of oligodendrocyte development in the vertebrate CNS. Prog Neurobiol 67:451-467.

Miyazawa K, Himi T, Garcia V, Yamagishi H, Sato S, Ishizaki Y (2000) A 
role for $\mathrm{p} 27 / \mathrm{Kip} 1$ in the control of cerebellar granule cell precursor proliferation. J Neurosci 20:5756-5763.

Ohnuma S, Philpott A, Wang K, Holt CE, Harris WA (1999) p27Xic1, a Cdk inhibitor, promotes the determination of glial cells in Xenopus retina. Cell 99:499-510.

Pagliuca A, Gallo P, De Luca P, Lania L (2000) Class A helix-loop-helix proteins are positive regulators of several cyclin-dependent kinase inhibitors' promoter activity and negatively affect cell growth. Cancer Res 60:1376-1382.

Park H, Mehta A, Richardson JS, Appel B (2002) olig2 is required for zebrafish primary motor neuron and oligodendrocyte development. Dev Biol 248:356-368.

Park H, Shin J, Appel B (2004) Spatial and temporal regulation of ventral spinal cord precursor specification by Hedgehog signaling. Development 131:5959-5969.

Park H-C, Appel B (2003) Delta-Notch signaling regulates oligodendrocyte specification. Development 130:3747-3755.

Scheer N, Campos-Ortega JA (1999) Use of the Gal4-UAS technique for targeted gene expression in the zebrafish. Mech Dev 80:153-158.

Scheer N, Groth A, Hans S, Campos-Ortega JA (2001) An instructive function for Notch in promoting gliogenesis in the zebrafish retina. Development 128:1099-1107.

Scheer N, Riedl I, Warren JT, Kuwada JY, Campos-Ortega JA (2002) A quantitative analysis of the kinetics of Gal4 activator and effector gene expression in the zebrafish. Mech Dev 112:9-14.

Schier AF, Neuhauss SC, Harvey M, Malicki J, Solnica-Krezel L, Stainier DY, Zwartkruis F, Abdelilah S, Stemple DL, Rangini Z, Yang H, Driever W (1996) Mutations affecting the development of the embryonic zebrafish brain. Development 123:165-178.

Shin J, Park HC, Topczewska JM, Mawdsley DJ, Appel B (2003) Neural cell fate analysis in zebrafish using olig2 BAC transgenics. Methods Cell Sci 25:7-14.
Shoji W, Yee CS, Kuwada JY (1998) Zebrafish semaphorin Zla collapses specific growth cones and alters their pathway in vivo. Development 125:1275-1283.

Sprague J, Doerry E, Douglas S, Westerfield M (2001) The Zebrafish Information Network (ZFIN): a resource for genetic, genomic and developmental research. Nucleic Acids Res 29:87-90.

Thermes V, Grabher C, Ristoratore F, Bourrat F, Choulika A, Wittbrodt J, Joly JS (2002) I-SceI meganuclease mediates highly efficient transgenesis in fish. Mech Dev 118:91-98.

Turner DL, Weintraub H (1994) Expression of achaete-scute homolog 3 in Xenopus embryos converts ectodermal cells to a neural fate. Genes Dev 8:1434-1447.

Vernon AE, Devine C, Philpott A (2003) The cdk inhibitor p27Xicl is required for differentiation of primary neurones in Xenopus. Development 130:85-92.

Wallace K, Liu TH, Vaessin H (2000) The pan-neural bHLH proteins DEADPAN and ASENSE regulate mitotic activity and cdk inhibitor dacapo expression in the Drosophila larval optic lobes. Genesis 26:77-85.

Wang S, Sdrulla AD, diSibio G, Bush G, Nofziger D, Hicks C, Weinmaster G, Barres BA (1998) Notch receptor activation inhibits oligodendrocyte differentiation. Neuron 21:63-75.

Westerfield M (2000) The zebrafish book. Eugene, OR: University of Oregon.

Wettstein DA, Turner DL, Kintner C (1997) The Xenopus homolog of Drosophila Suppressor of Hairless mediates Notch signaling during primary neurogenesis. Development 124:693-702.

Zezula J, Casaccia-Bonnefil P, Ezhevsky SA, Osterhout DJ, Levine JM, Dowdy SF, Chao MV, Koff A (2001) p21cip1 is required for the differentiation of oligodendrocytes independently of cell cycle withdrawal. EMBO Rep 2:27-34.

Zhu L, Skoultchi AI (2001) Coordinating cell proliferation and differentiation. Curr Opin Genet Dev 11:91-97. 